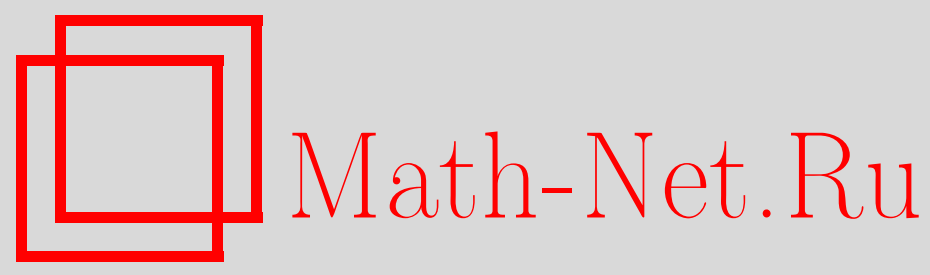

С. В. Пчелинцев, Многообразия разрешимых индекса 2 альтернативных алгебр над полем характеристики 3, Матем. заметки, 1999, том 66, выпуск 4, 556-566

DOI: https://doi.org/10.4213/mzm3988

Использование Общероссийского математического портала Math-Net.Ru подразумевает, что вы прочитали и согласны с пользовательским соглашением http://www . mathnet.ru/rus/agreement

Параметры загрузки:

IP: 54.162 .127 .20

26 апреля 2023 г., 14:37:50

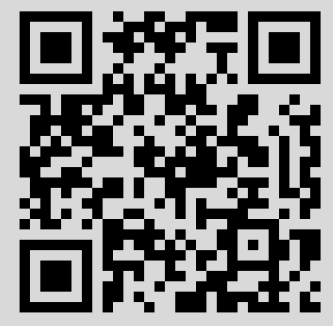




\title{
МНОГООБРАЗИЯ РАЗРЕШИМЫХ ИНДЕКСА 2 АЛЬТЕРНАТИВНЫХ АЛГЕБР НАД ПОЛЕМ ХАРАКТЕРИСТИКИ 3
}

\section{С. В. Пчелинцев}

\begin{abstract}
Изучаются подмногообразия многообразия $\mathrm{Alt}_{2}$ разрешимых индекса 2 альтернативных алгебр над произвольньм полем характеристики 3 . На языке тождеств выделены основные типы таких многообразий и установлены включения между ними. Основным результатом является
\end{abstract}

Теорема. Топологический ранг многообразия $\mathrm{Alt}_{2}$ разрешимых индекса 2 альтернативных алгебр над произвольным полем характеристики 3 равен 5.

Библиография: 6 названий.

Целью данной заметки является доказательство следующей теоремы.

ТеоремА. Топологический ранг многообразия $\mathrm{Alt}_{2}$ разрешимых индекса 2 альтернативных алгебр над произвольным полем характеристики 3 равен 5.

Известно [1], что многообразие $\mathrm{Alt}_{2}$ над полем характеристики, отличной от 2 и 3 , имеет два ненильпотентных подмногообразия. Отсюда, в частности, вытекает, что топологический ранг многообразия $\mathrm{Alt}_{2}$ равен 2. Анализ доказательств показьвает, что эти результаты справедливы и в случае поля характеристики 2.

Напомним, что из теоремы Ю. А. Медведева [2] о шпехтовости многообразия алгебр, удовлетворяющих двучленному тождеству, вытекает шпехтовость многообразий разрешимых индекса 2 алгебр, близких к ассоциативньм, в частности, многообразия Alt 2 . Строение решетки подмногообразий многообразия $\mathrm{Alt}_{2}$ над полем характеристики 0, в котором разрешимо уравнение $t^{2}+t+1=0$, описано А. В. Ильтяковым [3].

Данная работа состоит из трех параграфов. В 11 приведены основные определения и доказаны предварительные результаты. В $§ 2$ выделены основные типы подмногообразий в $\mathrm{Alt}_{2}$ и доказана оценка сверху для топологического ранга. Последний $§ 3$ посвящен построению необходимых примеров, существование которых дает оценку снизу для топологического ранга.

Работа выполнена при финансовой поддержке Российского фонда фундаментальных исследований, грант № 97-01-00785. 


\section{§1. Предварительные результаты}

1. Пространство полилинейных многочленов. Термин “алгебра" будет означать в дальнейшем, если не оговорено противное, разрешимую индекса 2 альтернативную алгебру над произвольным полем $\Phi$ характеристики 3.

Заметим, что поскольку в разрешимой индекса 2 альтернативной алгебре всякий одночлен, имеюший по некоторой переменной степень не меньшую 3 , нулевой, то любое многообразие разрешимых индекса 2 альтернативных алгебр определяется однородными тождествами степени не вьше 2 по каждой переменной и, следовательно, является однородным [4]. Поскольку характеристика поля равна 3, то всякое многообразие разрешимых индекса 2 альтернативных алгебр может быть задано некоторой системой полилинейных тождеств (многочленов); причем будем считать, не оговаривая этого специально, что каждое тождество степени $n$ зависит от переменных из множества $X_{n}=\left\{x_{1}, x_{2}, \ldots, x_{n}\right\}$.

Пусть $M$ - произвольное многообразие разрешимых индекса 2 альтернативных алгебр; $A=F_{M}[X]$ - свободная $M$-алгебра с множеством $X=\left\{x_{1}, x_{2}, \ldots\right\}$ свободньх порождающих.

ОПРЕДЕЛЕНИЕ [1]. Правильными словами алгебры $A$ от переменных из множества $X_{n}(n \geqslant 4)$ называются полилинейные одночлены:

a) $\left(x_{1} x_{j}\right) R\left(k_{1}\right) \cdots R\left(k_{n-2}\right), k_{1}<k_{2}<\cdots<k_{n-2}$;

б) $\left(x_{j} x_{1}\right) R\left(k_{1}\right) \cdots R\left(k_{n-2}\right), k_{1}<k_{2}<\cdots<k_{n-2}$;

в) $\left(x_{2} x_{3}\right) R(1) \cdots R(n)$;

где $R(k)$ - оператор правого умножения на элемент $x_{k}$.

Регулярным словом алгебры $A$ от переменных из $X_{n}$ назьвается слово

г) $\left(x_{3}\left(x_{1} x_{2}\right)\right) R(4) \cdots R(n)$.

ЛЕмма 1 [1]. Пространство $P_{n}(A)$ полилинейных одночленов над $X_{n}$ линейно порождается правильными и регулярными словами алгебры $A$.

Введем вспомогательные функции, кососимметрические по $y, z$ :

$$
\{x, y, z\}=(x \circ y) z-(x \circ z) y-(x, y, z), \quad[x, y, z]=[x, y] z-[x, z] y,
$$

где $x \circ y=x y+y x$ и $[x, y]=x y-y x-$ йорданово произведение и коммутатор соответственно.

Нам потребуется некоторая модификация системы правильных и регулярньх слов, которая также порождает пространство $P_{n}(A)$.

ЛЕмма 2. Пространство $P_{n}(A)(n \geqslant 4)$ линейно порождается әлементами вида, которые в дальнейшем называются базисными словами:

1) $\left(x_{1} \circ x_{i}\right) \rho_{i}, i \geqslant 4$

2) $\left[x_{1}, x_{i}\right] \rho_{i}, i \geqslant 4$

3) $\left(x_{1} \circ x_{2}\right) \rho_{2}$;

4) $\left[x_{1}, x_{2}\right] \rho_{2}$;

5) $\left\{x_{1}, x_{2}, x_{3}\right\} \eta$;

6) $\left[x_{1}, x_{2}, x_{3}\right] \eta$;

7) $\left(x_{1}, x_{2}, x_{3}\right) \eta$;

8) $\left[x_{1},\left[x_{2}, x_{3}\right]\right] \eta$; 
әде $\rho_{i}=R(2) \cdots R^{\wedge}(i) \cdots R(n)$ - операторное слово, в котором отсутствует опеpamop $R(i), \eta=R(4) R(5) \cdots R(n)$.

ДокАЗАТЕЛЬСТво. Из определения йорданова произведения и коммутатора над полем характеристики 3 вытекает равенство $x y=2 x \circ y+2[x, y]$, следовательно, правильные слова типа а) и б) при $j \neq 3$ представимы в виде линейной комбинации слов типа 1 ) и 2). Обозначим через $V$ пространство, порожденное элементами типа 3$)-8$ ) над $X_{4}$; а для многочленов $x, y$ над $X_{3}$ будем писать $x \equiv y$, если $(x-y) R(4) \in V$.

Ясно, что

$$
\left(x_{2} x_{1}\right) x_{3} \equiv\left(x_{1} x_{2}\right) x_{3} \equiv 0 .
$$

Из определения функций $\left\{x_{1}, x_{2}, x_{3}\right\}$ и $\left[x_{1}, x_{2}, x_{3}\right]$ имеем

$\left(x_{1} \circ x_{3}\right) x_{2}=\left(x_{1} \circ x_{2}\right) x_{3}-\left\{x_{1}, x_{2}, x_{3}\right\}-\left(x_{1}, x_{2}, x_{3}\right), \quad\left[x_{1}, x_{3}\right] x_{2}=\left[x_{1}, x_{2}\right] x_{3}-\left[x_{1}, x_{2}, x_{3}\right]$, т.е.

$$
\left(x_{3} x_{1}\right) x_{2} \equiv\left(x_{1} x_{3}\right) x_{2} \equiv 0 .
$$

Таким образом, из сравнений (1), (2) и замечания, сделанного в самом начале доказательства, вытекает, что все правильные слова типа а) и б) представимы в виде линейной комбинации базисньх слов. Значит, в силу леммы 1 достаточно проверить, что справедливы сравнения $v:=\left(x_{2} x_{3}\right) x_{1} \equiv 0, w:=x_{3}\left(x_{1} x_{2}\right) \equiv 0$.

Применяя сравнения (1), (2) и кососимметричность ассоциатора по всем переменным, получаем

$$
w=x_{3}\left(x_{1} x_{2}\right)=\left(x_{3} x_{1}\right) x_{2}-\left(x_{3}, x_{1}, x_{2}\right)=\left(x_{3} x_{1}\right) x_{2}-\left(x_{1}, x_{2}, x_{3}\right) \equiv 0
$$

аналогично,

$$
\begin{aligned}
& x_{1}\left(x_{2} x_{3}\right)=\left(x_{1} x_{2}\right) x_{3}-\left(x_{1}, x_{2}, x_{3}\right) \equiv 0 \\
& x_{1}\left(x_{3} x_{2}\right)=\left(x_{1} x_{3}\right) x_{2}-\left(x_{1}, x_{3}, x_{2}\right) \equiv 0 .
\end{aligned}
$$

Итак, осталось проверить, что $v \equiv 0$. Ввиду (3) имеем

$$
\begin{aligned}
v & =\left(x_{2} x_{3}\right) x_{1}=\left[x_{2} x_{3}, x_{1}\right]+x_{1}\left(x_{2} x_{3}\right) \equiv\left[x_{2} x_{3}, x_{1}\right] \\
& =2\left[\left[x_{2}, x_{3}\right], x_{1}\right]+2\left[x_{2} \circ x_{3}, x_{1}\right] \equiv 2\left[x_{2} \circ x_{3}, x_{1}\right],
\end{aligned}
$$

т.е.

$$
v \equiv 2\left[x_{2} \circ x_{3}, x_{1}\right]
$$

Поскольку в разрешимой индекса 2 альтернативной алгебре справедливо тождество

$$
\left\{\left(x_{2} \circ x_{3}\right) x_{1}+\left(x_{3} \circ x_{1}\right) x_{2}+\left(x_{1} \circ x_{2}\right) x_{3}\right\} x_{4}=0
$$

получаем ввиду $(3),(4),(6),(1),(2)$

$\left[x_{2} \circ x_{3}, x_{1}\right]=\left(x_{2} \circ x_{3}\right) x_{1}-x_{1}\left(x_{2} \circ x_{3}\right) \equiv\left(x_{2} \circ x_{3}\right) x_{1} \equiv-\left(x_{3} \circ x_{1}\right) x_{2}-\left(x_{1} \circ x_{2}\right) x_{3} \equiv 0$.

Сопоставляя последнее сравнение с (5), получаем $v \equiv 0$. 
2. Свойства функций $\{x, y, z\},[x, y, z]$.

Лемма 3. В алгебре А справедливы соотношения:

a) $\{x, x, y\} \in \operatorname{Ann} A$;

б) $[x, x, y]=\left[x^{2}, y\right]-[x,[x, y]]$.

ДокАЗАТЕЛЬСтво. Имеем на основании (6)

$\{x, x, y\} z=\{(x \circ x) y-(x \circ y) x\} z=\{-2(x \circ y) x-(x \circ y) x\} z=\{-3(x \circ y) x\} z=0$,

$[x, x, y]=[x, x] y-[x, y] x=2[x, y] x=[x, y] \circ x+[[x, y], x]=\left[x^{2}, y\right]+[[x, y], x]$.

Лемма 4. Пусть $w \in A^{2}$. Тогда в алгебре А справедливь равенства:

a) $[[w, y], z]=0$;

б) $\{w, y, z\}=[w, y] z$

в) $[w, y, z]=2[w, y] z$

г) $\{x, y, w\}=[x, y, w]=[w, x] y$.

ДокАЗАТЕЛЬСтво. Заметим, что в силу тождеств альтернативности и центрального тождества Муфанг $(a b)(c a)=a(b c) a$ верно равенство $w T(x) T(x)=0$, в частности, функция $w T(x) T(y)$ кососимметрическая по переменным $x$ и $y$. Тогда, используя это свойство, последовательно получаем:

a) $\quad[[w, x], y]=(w x-x w) y-y(w x-x w) \equiv(w x) y+(y w) x-y(w x)+y(x w)$

$$
\equiv(w, x, y)+(y, w, x)-(y, x, w)=3(w, x, y)=0,
$$

б) $\{w, y, z\}=(w \circ y) z-(w \circ z) y-(w, y, z)=2(w \circ y) z-(w, y, z)$

$=(2 w y+2 y w-w y) z=(w y-y w) z=[w, y] z$,

в) $[w, y, z]=[w, y] z-[w, z] y=2[w, y] z$,

г) $\{x, y, w\}=(x \circ y) w-(x \circ w) y-(x, y, w)=2(w \circ x) y-(w, x, y)$

$=(2 w x+2 x w-w x) y=(w x-x w) y=[w, x] y$,

$[x, y, w]=[x, y] w-[x, w] y=-[x, w] y=[w, x] y$.

\section{§ 2. Оценка топологического ранга сверху}

1. Основные тождества разрешимых индекса 2 альтернативных алгебр. В дальнейших рассмотрениях принципиальную роль играют следующие тождества и определяемые ими многообразия, обозначение которых указано в скобках:

a) тождество центральной коммутативности степени $n$

$$
\left(\mathbf{Z C}_{n}\right) \quad\left[\left[x_{2}, x_{3}\right], x_{1}\right] R(4) R(5) \cdots R(n) ;
$$

б) тождество центральной антикоммутативности степени $n$

$$
\left(\mathbf{Z A}_{n}\right) \quad\left[x_{1} \circ x_{2}, x_{3}\right] R(4) R(5) \cdots R(n) ;
$$

в) тождество коммутативности степени $n$

$$
\left(\mathbf{C o m}_{n}\right) \quad\left[x_{1}, x_{2}\right] R(3) R(4) \cdots R(n) ;
$$

г) тождество антикоммутативности степени $n$

$$
\left(\mathbf{A C}_{n}\right) \quad\left(x_{1} \circ x_{2}\right) R(3) R(4) \cdots R(n) .
$$


ЗАмЕчАниЕ. Поскольку антикоммутативная альтернативная алгебра нильпотентна, йорданово произведение в тождествах $\left(\mathbf{Z} \mathbf{A}_{n}\right),\left(\mathbf{A C}_{n}\right)$ можно заменить на обычное произведение, увеличив, если необходимо, число $n$.

Для произвольных элементов $a, b \in A^{2}$ будем писать

$$
a \equiv b, \quad \text { если }(\exists n)\left(\forall t_{1}, \ldots, t_{2 n} \in X\right) \quad(a-b) R\left(t_{1}\right) \cdots R\left(t_{2 n}\right)=0 .
$$

Поскольку оператор $R(s) R(t)$ является центральным элементом в алгебре $A^{*}$ умножений, действующих на идеале $A^{2}$, то введенное отношение эквивалентности сохраняется при умножении обеих частей сравнения на произвольньй элемент.

В дальнейшем нам потребуются следующие два тождества, вьполняющиеся в произвольной альтернативной алгебре характеристики 3 (см., например, [4]):

$$
\begin{aligned}
{[a b, c] } & =a[b, c]+[a, c] b, \\
(a \circ b) \circ c-(a \circ c) \circ b & =[a,[b, c]]+2(a, b, c) .
\end{aligned}
$$

Лемма 5. Пусть алгебра $A$ ненильпотентна и для любых $а, x \in A, w \in A^{2}$ справедливо сравнение

$$
\alpha[x \circ w, a]+\beta(x \circ w) a \equiv 0
$$

Тогда $\beta=0$.

ДокАЗАТЕЛЬСТво. Из условия вытекает $\alpha[[x \circ w, a], b]+\beta[(x \circ w) a, b] \equiv 0$. Отсюда в силу леммы 4 имеем $\beta[(x \circ w) a, b] \equiv 0$, значит согласно тождеству $(7) \beta[x \circ w, b] a \equiv 0$, или $\beta[x \circ w, b] \equiv 0$. Умножая сравнение $(9)$ на $\beta$ и учитьвая $\beta[x \circ w, a] \equiv 0$, имеем сравнение $\beta^{2}(x \circ w) a \equiv 0$. Так как в силу тождества (8) сравнение $x \circ w \equiv 0$ влечет нильпотентность алгебры $A$, то $\beta=0$.

Лемма 6. Пусть свободная $M$-алгебра А удовлетворяет тохсдеству

$$
\sum_{i \geqslant 4}\left(\alpha_{i} x_{1} \circ x_{i}+\beta_{i}\left[x_{1}, x_{i}\right]\right) \rho_{i}+f=0
$$

где $f$ - линейная комбинация базисных слов типа 3)-8) из леммы 2. Тогда

а) если алгебра $A$ ненильпотентна, то $\alpha_{i}=0$;

б) если алгебра $A$ не является иентрально антикоммутативной, то $\alpha_{i}=0$, $\beta_{i}=0$.

ДокАЗАтЕльство. Допустим, что хотя бы один из скаляров $\alpha_{p}, \beta_{p}$ отличен от 0 (при фиксированном $p$ ). Подставим $x_{p}=w \in A^{2}$ в тождество $(*)$. Тогда в силу тождества разрешимости индекса 2 получим

$$
\alpha_{p}(w \circ x) \equiv \beta_{p}[w, x]
$$

Утверждение а) следует из леммы 5.

Если же алгебра $A$ не является центрально антикоммутативной, то она ненильпотентна, $\alpha_{p}=0$. Но тогда из (10) следует $\beta_{p}=0$. 
2. Базис свободной разрешимой индекса 2 альтернативной алгебры. Напомним, что базисным.м словами алгебры $A$ muпа 1)-8) мы назьваем порождающую совокупность линейного пространства $P_{n}(A)$, указанную в лемме 2 .

Лемма 7. Базисные слова свободной алгебры $A=F_{M}[X]$ степени $n \geqslant 4$ линейно зависимы тогда и только тогда, когда многообразие $M$ иентрально коммутативно, m.e. $M \subseteq \mathbf{Z C}=\cup \mathbf{Z} \mathbf{C}_{i}$.

ДоказАтельство. Допустим, что базисные слова алгебры $A$ степени $n \geqslant 4$ линейно зависимы и алгебра $A$ не является центрально коммутативной. Тогда в алгебре $A$ справедливо тождество $(*)$, которое в силу леммы 6 принимает вид

$\alpha\left(x_{1} \circ x_{2}\right) x_{3}+\beta\left[x_{1}, x_{2}\right] x_{3}+\gamma\left\{x_{1}, x_{2}, x_{3}\right\}+\delta\left[x_{1}, x_{2}, x_{3}\right]+\lambda\left(x_{1}, x_{2}, x_{3}\right)+\mu\left[x_{1},\left[x_{2}, x_{3}\right]\right] \equiv 0$,

причем хотя бы один из скаляров в этом сравнении отличен от 0 .

Полагая $x_{3}=[[a, b], c]$, на основании леммы 4 получим $\lambda\left(x_{1}, x_{2},[[a, b], c]\right) \equiv 0$, откуда $\lambda[[a, b], c] \equiv 0$. Поскольку алгебра $A$ не является центрально коммутативной, то $\lambda=0$. Следовательно, алгебра $A$ удовлетворяет тождеству

$$
\alpha\left(x_{1} \circ x_{2}\right) x_{3}+\beta\left[x_{1}, x_{2}\right] x_{3}+\gamma\left\{x_{1}, x_{2}, x_{3}\right\}+\delta\left[x_{1}, x_{2}, x_{3}\right]+\mu\left[x_{1},\left[x_{2}, x_{3}\right]\right] \equiv 0 .
$$

Полагая $x_{3}=w \in A^{2}$ в тождестве (11), получим ввиду леммы $4(\gamma+\delta)\left[w, x_{1}\right] x_{2} \equiv 0$, откуда $\gamma+\delta \equiv 0$. Тем самьм, тождество (11) принимает вид

$$
\alpha\left(x_{1} \circ x_{2}\right) x_{3}+\beta\left[x_{1}, x_{2}\right] x_{3}+\gamma\left(\left\{x_{1}, x_{2}, x_{3}\right\}-\left[x_{1}, x_{2}, x_{3}\right]\right)+\mu\left[x_{1},\left[x_{2}, x_{3}\right]\right] \equiv 0 .
$$

Пусть $x_{1}=x_{2}=x, x_{3}=y$. Тогда в силу леммы 3 имеем

$$
2 \alpha x^{2} y-\gamma\left(\left[x^{2}, y\right]-[x,[x, y]]\right)+\mu[x,[x, y]] \equiv 0 .
$$

Линеаризуем последнее тождество подстановкой $x \mapsto w$ и воспользуемся леммой 4 :

$$
2 \alpha(x \circ w) y-\gamma[x \circ w, y] \equiv 0 .
$$

Отсюда в силу леммы $5 \alpha=0, \gamma=0$, а из (11) вытекает $\mu=0$, поскольку было доказано, что все остальные скаляры в (11) нулевые.

ЗАмечАниЕ. Из примера 1 (см. § 3 ниже) следует, что система базисных слов свободной разрешимой индекса 2 альтернативной алгебры образует аддитивный базис пространства полилинейных многочленов степени, не меньшей 4.

3. Заключительную часть этого параграфа посвятим получению оценок топологического ранга сверху.

ПРЕДЛОЖЕНИЕ. Справедливы неравенства:

1) $r_{t}\left(\mathrm{Alt}_{2}\right) \leqslant r_{t}(\mathbf{Z C})+1$

2) $r_{t}(\mathbf{Z C}) \leqslant r_{t}(\mathbf{Z A})+1$, әде $\mathbf{Z A}=\cup \mathbf{Z A} \mathbf{A}_{i}$;

3) $r_{t}(\mathbf{Z A}) \leqslant r_{t}(\mathbf{C o m})+1$, əде $\mathbf{C o m}=\cup \mathbf{C o m}_{i}$;

4) $r_{t}(\mathbf{C o m}) \leqslant r_{t}(\mathbf{A C})+1$, дде $\mathbf{A C}=\cup \mathbf{A C}_{i}$. 
1) Лемма 7 может быть переформулирована на топологическом языке. Для этого напомним основные понятия [1], связанные с введением топологии на решетке $(M)$ подмногообразий шпехтова многообразия $M$.

Пусть $V$ - конечнобазируемое многообразие, $V \subsetneq W$. Размерностью $\operatorname{dim}_{W} V$ многообразия $V$ относительно $W$ называется наименьшее число $n$, обладающее свойством: существует конечная система тождеств $f_{1}, \ldots, f_{s}$, выделяющая $V$ из $W$, т.е. $\left\langle f_{1}, \ldots, f_{s}\right\rangle^{T}+T(W)=T(V)$, такая, что ни одно из тождеств этой системы не вьполняется в $W$ и $n=\max \left\{\operatorname{deg} f_{1}, \ldots, \operatorname{deg} f_{s}\right\}$. Под размерностью $\operatorname{dim} V$ многообразия $V$ понимается размерность $V$ относительно многообразия всех алгебр.

Пусть $M$ - шпехтово многообразие; $\Omega \subset \wp(M)$. Множество $\Omega$ назьвается конечномерным, если размерности всех многообразий из множества $\Omega$ ограничены в совокупности.

Для любого $W$ из ю( $M)$ введем множества

$$
\stackrel{\circ}{U}_{n}(W)=\left\{V \subset W \mid \operatorname{dim}_{W} V \geqslant n\right\}, \quad U_{n}(W)=\stackrel{\circ}{U}_{n}(W) \cup\{W\}
$$

Считая систему множеств $\Sigma=\left\{U_{n}(W) \mid W \in \wp(M), n \in \mathbb{N}\right\}$ базой окрестностей, наделяем $\wp(M)$ некоторой топологией; пусть $\Omega$ является топологическим подпространством пространства $\wp(M)$. Поскольку $M$ шшехтово, любая убывающая цепочка многообразий $V_{1} \supset V_{2} \supset \cdots \supset V_{n} \supset \cdots$ из $\Omega$ стабилизируется, следовательно, всякий минимальный элемент множества $\Omega$ является изолированной точкой в пространстве $\Omega$. Обозначая через $\Omega^{\prime}$ множество предельных точек пространства $\Omega$, имеем $\Omega^{\prime} \subsetneq \Omega$. Tопологическим рангом $r_{t}(\Omega)$ пространства $\Omega$ называется число $r$ такое, что $\widehat{\Omega}^{(r-1)} \neq \varnothing$ и $\Omega^{(r)}=\varnothing$. Топологическим рангом $r_{t}(M)$ многообразия $M$ называется топологический ранг пространства $\wp(M)$, т.е. $r_{t}(M)=r_{t}(\wp(M))$.

ЛЕмма 8 [1]. Пусть $F \subseteq E \subseteq \wp(M)$ и для любого многообразия $N \in E$ существует натуральное число $n$ такое, что $\stackrel{\circ}{U}_{n}(N) \subseteq F$. Тогда $r_{t}(E) \leqslant r_{t}(F)+1$.

Таким образом, из леммы 7 вытекает неравенство 1).

2) В силу леммы 8 для доказательства неравенства 2) достаточно понять, что если в свободной центрально коммутативной алгебре $A$ выполнено нетривиальное тождество

$$
\sum_{i \geqslant 4}\left(\alpha_{i} x_{1} \circ x_{i}+\beta_{i}\left[x_{1}, x_{i}\right]\right) \rho_{i}+f=0
$$

где $f$ - линейная комбинация базисных слов типа 3$)-7)$; то алгебра $A$ центрально антикоммутативна. Предположим от противного, что алгебра $A$ не является центрально антикоммутативной. Тогда по лемме 6 все скаляры $\alpha_{i}, \beta_{i}$ нулевые, и тождество принимает вид

$$
\alpha\left(x_{1} \circ x_{2}\right) x_{3}+\beta\left[x_{1}, x_{2}\right] x_{3}+\gamma\left\{x_{1}, x_{2}, x_{3}\right\}+\delta\left[x_{1}, x_{2}, x_{3}\right]+\lambda\left(x_{1}, x_{2}, x_{3}\right) \equiv 0,
$$

причем хотя бы один из скаляров отличен от 0.

Рассуждаем аналогично доказательству леммы 7. Полагая $x_{3}=[a, b]$, на основании леммы 4 получим $\lambda\left(x_{1}, x_{2},[a, b]\right) \equiv 0$, т.е. $\lambda[a, b] \equiv 0$. Поскольку алгебра $A$ не является 
центрально антикоммутативной, то $\lambda=0$. Следовательно, алгебра $A$ удовлетворяет тождеству

$$
\alpha\left(x_{1} \circ x_{2}\right) x_{3}+\beta\left[x_{1}, x_{2}\right] x_{3}+\gamma\left\{x_{1}, x_{2}, x_{3}\right\}+\delta\left[x_{1}, x_{2}, x_{3}\right] \equiv 0 .
$$

Полагая $x_{3}=w \in A^{2}$ в тождестве $(13)$, получим ввиду леммы $4(\gamma+\delta)\left[w, x_{1}\right] \equiv 0$, откуда $\gamma+\delta \equiv 0$, а тождество (13) принимает вид

$$
\alpha\left(x_{1} \circ x_{2}\right) x_{3}+\beta\left[x_{1}, x_{2}\right] x_{3}+\gamma\left(\left\{x_{1}, x_{2}, x_{3}\right\}-\left[x_{1}, x_{2}, x_{3}\right]\right) \equiv 0 .
$$

Пусть $x_{1}=x_{2}=x, x_{3}=y$. Тогда в силу леммы 3 и центральной коммутативности алгебры $A$ имеем $2 \alpha x^{2} y-\gamma\left[x^{2}, y\right] \equiv 0$. Линеаризуем последнее тождество подстановкой $x \mapsto w: 2 \alpha(x \circ w) y-\gamma[x \circ w, y] \equiv 0$. Отсюда в силу леммы $5 \alpha=0, \gamma=0$. Но тогда из (13) вытекает, что $\beta=0$, т.е. все скаляры в сравнении (12) нулевые. Полученное противоречие завершает доказательство.

3) Приступим к обоснованию неравенства 3 ).

ЛЕмма 9. Пусть $A$ - свободная иентрально антикоммутативная алгебра степени $n_{0}$. Тогда пространство $P_{n}(A)\left(n \geqslant n_{0}+1\right)$ линейно порожсдается базисными словами типа 1), 3), 4) и 7).

ДокАЗАТЕЛЬСтво. Проверим сначала, что базисные слова степени, большей $n_{0}$, линейно выражаются через указанные базисные слова типа 1)-4), 7). Обозначим через $V$ линейное подпространство в $P_{n}(A)$, порожденное базисньми словами указанных типов. Будем писать $x \equiv y$, если $(x-y) \rho \in V$, где $\rho$ - произвольное $R$-слово длины $n-3$. Заметим, что тождество центральной антикоммутативности степени $n_{0}$ влечет тождество центральной коммутативности степени $n_{0}+1$.

Докажем сначала сравнения

$$
\left\{x_{1}, x_{2}, x_{3}\right\} \equiv\left[x_{1}, x_{2}, x_{3}\right] \equiv\left[x_{1},\left[x_{2}, x_{3}\right]\right] \equiv 0 .
$$

Заметим, что тождество центральной антикоммутативности степени $n_{0}$ влечет тождество центральной коммутативности степени $n_{0}+1$, значит справедливо сравнение $\left[x_{1},\left[x_{2}, x_{3}\right]\right] \equiv 0$. Далее, поскольку верно равенство $\left(x_{1} \circ x_{2}\right) \circ x_{3}=2\left(x_{1} \circ x_{2}\right) x_{3}$, из тождества (8) имеем $\left(x_{1} \circ x_{2}\right) x_{3}-\left(x_{1} \circ x_{2}\right) x_{3} \equiv\left(x_{1}, x_{2}, x_{3}\right)$, т.е. $\left\{x_{1}, x_{2}, x_{3}\right\} \equiv 0$. Наконец, в силу тождества (7) и центральной антикоммутативности

$$
\left[x_{1}, x_{2}, x_{3}\right]=\left[x_{1}, x_{2}\right] x_{3}-\left[x_{1}, x_{3}\right] x_{2}=2\left[x_{1}, x_{2}\right] x_{3} \equiv 0 .
$$

Для завершения доказательства леммы осталось понять, что слова типа 2) линейно выражаются через слова типа 4). В силу тождества (7) имеем

$$
\left[x_{1}, x_{i}\right] x_{2}=\left[x_{1}, x_{i} x_{2}\right]-x_{i}\left[x_{1}, x_{2}\right]=\left[x_{1}, x_{2} x_{i}\right]-\left[x_{i},\left[x_{1}, x_{2}\right]\right]-\left[x_{1}, x_{2}\right] x_{i}
$$

откуда в силу центральной антикоммутативности и вытекает требуемое.

Теперь мы можем перейти именно к доказательству неравенства 3). Для этого в силу лемм 8 и 9 достаточно понять, что если в свободной центрально антикоммутативной алгебре $A$ вьполнено нетривиальное тождество степени $\geqslant n_{0}$

$$
\sum_{i \geqslant 4} \alpha_{i}\left(x_{1} \circ x_{i}\right) \rho_{i}+\beta\left(x_{1} \circ x_{2}\right) \rho_{2}+\gamma\left[x_{1}, x_{2}\right] \rho_{2}+\delta\left(x_{1}, x_{2}, x_{3}\right) \eta=0,
$$


то алгебра $A$ коммутативна некоторой степени.

Предположим от противного, что алгебра $A$ не является коммутативной. Подставляя $x_{i}=w \in A^{2}(i \geqslant 4)$, получим $\alpha_{i}=0$, иначе алгебра $A$ была бы нильпотентна. Тогда в алгебре $A$ вьполнено тождество

$$
\beta\left(x_{1} \circ x_{2}\right) \rho_{2}+\gamma\left[x_{1}, x_{2}\right] \rho_{2}+\delta\left(x_{1}, x_{2}, x_{3}\right) \eta=0,
$$

причем хотя бы один из скаляров отличен от 0 . Полагая $x_{3}=w \in A^{2}$ в тождестве (14), получим $\delta=0$, и тождество (14) принимает вид $\beta\left(x_{1} \circ x_{2}\right) \rho_{2}+\gamma\left[x_{1}, x_{2}\right] \rho_{2}=0$. Полагая здесь $x_{2}=[a, b]$, получаем $\beta=0$, тогда и $\gamma=0$. Полученное противоречие завершает доказательство неравенства 3 ).

4) Пусть $A$ - свободная коммутативная алгебра степени $n_{0}$. Тогда пространство $P_{n}(A)\left(n \geqslant n_{0}\right)$ линейно порождается базисными словами типа 1$\left.\left.), 3\right), 7\right)$. Значит, для доказательства неравенства 4 ) достаточно понять, что если в алгебре $A$ выполнено нетривиальное тождество степени $\geqslant n_{0}$

$$
\sum_{i \geqslant 4} \alpha_{i}\left(x_{1} \circ x_{i}\right) \rho_{i}+\beta\left(x_{1} \circ x_{2}\right) \rho_{2}+\gamma\left(x_{1}, x_{2}, x_{3}\right) \eta=0,
$$

то алгебра $A$ нильпотентна.

Предположим от противного, что алгебра $A$ ненильпотентна. Подставляя $x_{i}=w \in A^{2}$ $(i \geqslant 4)$, получим $\alpha_{i}=0$. Следовательно, в алгебре $A$ выполнено тождество

$$
\beta\left(x_{1} \circ x_{2}\right) \rho_{2}+\gamma\left(x_{1}, x_{2}, x_{3}\right) \eta=0,
$$

причем хотя бы один из скаляров отличен от 0 . Полагая $x_{3}=w \in A^{2}$ и используя коммутативность, получим $\gamma=0$. Тогда $\beta\left(x_{1} \circ x_{2}\right) \rho_{2}=0$, откуда $\beta=0$. Полученное противоречие завершает обоснование неравенства 4).

Итак, топологический ранг многообразия $\mathrm{Alt}_{2}$ разрешимых индекса 2 альтернативных алгебр над полем характеристики 3 не превосходит 5 (см. неравенства 1)-4)).

\section{§3. Оценка топологического ранга снизу}

Для получения оценки топологического ранга снизу приведем четыре примера. Все необходимые разрешимые индекса 2 альтернативные алгебры над полем $\Phi$ характеристики 3 строятся в виде грассмановых оболочек подходящих супералгебр. Проверку альтернативности соответствующих супералгебр мы опускаем.

Пример 1. Пусть $A=A_{0}+A_{1}$ - супералгебра с базисом $a_{0}, b_{0}, x, a_{1}, b_{1}: A_{0}=$ $\Phi a_{0}+\Phi b_{0}, A_{1}=\Phi x+\Phi a_{1}+\Phi b_{1}$, и следующей таблицей умножения:

\begin{tabular}{|c|c|c|c|c|c|}
\hline & $x$ & $a_{0}$ & $b_{0}$ & $a_{1}$ & $b_{1}$ \\
\hline$x$ & $a_{0}$ & $b_{1}$ & $-a_{1}$ & $b_{0}$ & $-a_{0}$ \\
\hline$a_{0}$ & $a_{1}$ & 0 & 0 & 0 & 0 \\
\hline$b_{0}$ & $a_{1}+b_{1}$ & 0 & 0 & 0 & 0 \\
\hline$a_{1}$ & $a_{0}$ & 0 & 0 & 0 & 0 \\
\hline$b_{1}$ & $b_{0}-a_{0}$ & 0 & 0 & 0 & 0 \\
\hline
\end{tabular}


Заметим, что грассманова оболочка $G(A)$ построенной супералгебры является алгеброй Г. В. Дорофеева [5]. Алгебра $G(A)$ не является центрально коммутативной, поскольку $\left[[x, x]_{s}, x\right]_{s}=2\left[a_{0}, x\right]_{s}=2\left(a_{1}-b_{1}\right)$, где $[a, b]_{s}$ - суперкоммутатор,

$$
\left(a_{1}-b_{1}\right) x \cdot x=\left(a_{0}-\left(b_{0}-a_{0}\right)\right) x=-\left(a_{0}+b_{0}\right) x=-\left(a_{1}+\left(a_{1}+b_{1}\right)\right)=a_{1}-b_{1} .
$$

ПримеР 2 [6]. Пусть $A=A_{0}+A_{1}$ - супералгебра с базисом $a_{0}, b_{0}, x, a_{1}, b_{1}: A_{0}=$ $\Phi a_{0}+\Phi b_{0}, A_{1}=\Phi x+\Phi a_{1}+\Phi b_{1}$, и следующей таблицей умножения:

\begin{tabular}{|c|c|c|c|c|c|}
\hline & $x$ & $a_{0}$ & $b_{0}$ & $a_{1}$ & $b_{1}$ \\
\hline$x$ & 0 & $b_{1}$ & $a_{1}+b_{1}$ & $a_{0}-b_{0}$ & $-a_{0}$ \\
\hline$a_{0}$ & $a_{1}$ & 0 & 0 & 0 & 0 \\
\hline$b_{0}$ & $-b_{1}$ & 0 & 0 & 0 & 0 \\
\hline$a_{1}$ & $a_{0}$ & 0 & 0 & 0 & 0 \\
\hline$b_{1}$ & $-b_{0}$ & 0 & 0 & 0 & 0 \\
\hline
\end{tabular}

Проверим, что супералгебра $A$ удовлетворяет тождеству $\left[[a, b]_{s}, c\right]_{s}=0$. Как следует из таблицы умножения, любой ненулевой суперкоммутатор базисных элементов $\mathrm{c}$ точностью до знака совпадает с одним из элементов: $a_{0}+b_{0}, a_{1}-b_{1}$. Далее,

$$
\begin{aligned}
& {\left[a_{0}+b_{0}, x\right]_{s}=\left[a_{0}, x\right]_{s}+\left[b_{0}, x\right]_{s}=\left(a_{1}-b_{1}\right)+\left(-b_{1}-\left(a_{1}+b_{1}\right)\right)=-3 b_{1}=0,} \\
& {\left[a_{1}-b_{1}, x\right]_{s}=\left[a_{1}, x\right]_{s}-\left[b_{1}, x\right]_{s}=\left(a_{0}+\left(a_{0}-b_{0}\right)\right)-\left(-b_{0}-a_{0}\right)=3 a_{0}=0,} \\
& {\left[a_{0}+b_{0}, c\right]_{s}=\left[a_{1}-b_{1}, c\right]_{s}=0 \quad \text { для } c=a_{0}, b_{0}, a_{1}, b_{1} .}
\end{aligned}
$$

Поскольку $\left(a_{0} x\right) \cdot x=a_{1} x=a_{0},\left[a_{0}, x\right]_{s}=a_{1}-b_{1},\left(a_{1}-b_{1}\right) x \cdot x=\left(a_{0}+b_{0}\right) x=a_{1}-b_{1}$, то грассманова оболочка супералгебры $A$ является центрально коммутативной степени 3 , но не является центрально антикоммутативной никакой степени.

Пример 3. Пусть $A=A_{0}+A_{1}$ - супералгебра с базисом $a_{0}, x, a_{1}: A_{0}=\Phi a_{0}$, $A_{1}=\Phi x+\Phi a_{1}$, и следующей таблицей умножения:

\begin{tabular}{|c|c|c|c|}
\hline & $x$ & $a_{0}$ & $a_{1}$ \\
\hline$x$ & $a_{0}$ & $a_{1}$ & $-a_{0}$ \\
\hline$a_{0}$ & $a_{1}$ & 0 & 0 \\
\hline$a_{1}$ & $a_{0}$ & 0 & 0 \\
\hline
\end{tabular}

Заметим, что $A^{2}=\Phi a_{0}+\Phi a_{1},\left[a_{0}, x\right]_{s}=\left[a_{1}, x\right]_{s}=0$. Кроме того, $[x, x]_{s}=2 a_{0}$, $\left(a_{0} x\right) \cdot x=a_{1} x=a_{0}$, следовательно, грассманова оболочка супералгебры $A$ является центрально антикоммутативной степени 3 , но не удовлетворяет тождеству коммутативности никакой степени. 
Пример 4 [6]. Пусть $A=A_{0}+A_{1}$ - супералгебра с базисом $a_{0}, x, a_{1}: A_{0}=\Phi a_{0}$, $A_{1}=\Phi x+\Phi a_{1}$, и следующей таблицей умножения:

\begin{tabular}{|c|c|c|c|}
\hline & $x$ & $a_{0}$ & $a_{1}$ \\
\hline$x$ & 0 & $a_{1}$ & $-a_{0}$ \\
\hline$a_{0}$ & $a_{1}$ & 0 & 0 \\
\hline$a_{1}$ & $a_{0}$ & 0 & 0 \\
\hline
\end{tabular}

Заметим, что алгебра $A$ суперкоммутативна, но ненильпотентна: $\left(a_{0} x\right) \cdot x=a_{1} x=a_{0}$, следовательно, грассманова оболочка супералгебры $A$ является обычной коммутативной, но ненильпотентной алгеброй.

\section{СПИСОК ЦИТИРОВАННОЙ ЛИТЕРАТУРЫ}

[1] Пчелинцев С. В. Разрешимые индекса 2 многообразия алгебр // Матем. сб. 1981. Т. 115. С. $179-203$.

[2] Медведев Ю.А. Конечная базируемость многообразий с двучленным тождеством // Алгебра и логика. 1978. Т. 17. №6. С. 705-726.

[3] Ильтяков А. В. Решетка подмногообразий многообразия двухступенчато разрешимых альтернативных алгебр // Алгебра и логика. 1982. Т. 21. № 2. С. 170-177.

[4] Жевлаков К. А., Слинько А. М., Шестаков И. П., Ширшов А. И. Кольца, близкие к ассоциативным. М.: Наука, 1978.

[5] Дорофеев Г.В. Пример разрешимого, но не нильпотентного альтернативного кольца // УМН. 1960. Т. 15. №3. С. $147-150$.

[6] Шестаков И.П. Супералгебры и контрпримеры // Сиб. матем. ж. 1991. Т. 32. №6. C. $187-196$.

Московский городской педагогический университет 